\title{
PSR B1929+10 revisited in X-rays
}

\author{
A. Słowikowska ${ }^{1}$, L. Kuiper ${ }^{2}$, and W. Hermsen ${ }^{2}$ \\ 1 Nicolaus Copernicus Astronomical Center, Rabiańska 8, 87-100 Toruń, Poland \\ e-mail: aga@ncac.torun.pl \\ 2 SRON National Institute for Space Research, Sorbonnelaan 2, 3584 CA Utrecht, The Netherlands \\ e-mail: [L.M.Kuiper;W.Hermsen]@sron.nl
}

Received 2 March 2004 / Accepted 3 January 2005

\begin{abstract}
We performed timing and spectral analyses for PSR B1929+10, one of the oldest ( $\sim 10^{7}$ years) of the ordinary pulsars detected in X-rays, using archival ROSAT, ASCA and RXTE data. Pulsed emission was detected at a more than five sigma level for the combined ROSAT PSPC-B and previously unpublished HRI data. Our pulse profile is in agreement with that obtained by Yancopoulos et al. (1994, ApJ, 429, 832, ROSAT PSPC-B) but now with better statistics. The pulsed fraction in the ROSAT $\mathrm{X}$-ray band is $0.25 \pm 0.04$. The pulsed signal has also been detected in the ASCA GIS data $(0.5-5 \mathrm{keV})$ with a similar pulsed fraction of $0.36 \pm 0.11$. No significant timing signal is found in the RXTE PCA data $(>2 \mathrm{keV})$. We found that the combined ROSAT PSPC-B and ASCA GIS spectrum can satisfactorily be described by a power-law as well as by a double black-body model but not by a single black-body model or black-body plus power law model. Fitting the combined ROSAT/ASCA 0.1$10 \mathrm{keV}$ spectrum by a power-law model we obtain a photon index $\alpha$ of $2.54 \pm 0.12$ and a neutral hydrogen column density $N_{\mathrm{H}}$ towards the source of $9.8_{-1.0}^{+1.4} \times 10^{20} \mathrm{~cm}^{-2}$. For a double black-body fit our results are $T_{1}=2.0_{-0.05}^{+0.05} \times 10^{6} \mathrm{~K}, T_{2}=6.9_{-0.35}^{+0.23} \times 10^{6} \mathrm{~K}$ and $N_{\mathrm{H}}=4.4_{-1.1}^{+2.1} \times 10^{20} \mathrm{~cm}^{-2}$. In both cases the derived value of $N_{\mathrm{H}}$ is higher than that adopted in earlier works, but our result is fully consistent with the larger distance estimate of $331 \pm 10 \mathrm{pc}$ from parallax measurements combined with the hydrogen distribution measurements in the direction to the pulsar.
\end{abstract}

Key words. stars: neutron - pulsars: individual: PSR B1929+10

\section{Introduction}

PSR B1929+10 is one of the closest and oldest known ordinary pulsars detected in X-rays. The two latest investigations of this object in X-rays were based on ROSAT PSPC-B (Yancopoulos et al. 1994, hereafter Y94) and ASCA (Wang \& Halpern 1997, hereafter WH97) observations with exposure durations of $45 \mathrm{ks}$ and $54 \mathrm{ks}$, respectively. After background subtraction Y94 obtained a total of $420 \pm 25$ photons in the $0.1-2.0 \mathrm{keV}$ band, which corresponds to a luminosity of $1.2 \times 10^{30} \mathrm{ergs} \mathrm{s}^{-1}$ for a source distance of $250 \mathrm{pc}$, or to about $3 \times 10^{-4}$ of the pulsar's spin-down luminosity. Furthermore, folding the barycentered arrival times of the selected events, Y94 fitted the pulse shape using a sinusoid and this yielded a pulsed fraction of $0.28 \pm 0.10(0.1-2.0 \mathrm{keV})$.

The spectrum was fitted with a black-body yielding a temperature $T_{\infty} \sim 3.2 \times 10^{6} \mathrm{~K}$ and an emitting area with a radius of less than $40 \mathrm{~m}$, assuming an absorbing hydrogen column density of $1.5 \times 10^{20} \mathrm{~cm}^{-2}$. WH97 used ASCA data from October 1994 and fitted the spectrum of PSR B1929+10 over the $0.5-5.0 \mathrm{keV}$ range, also with a single black-body. The neutral hydrogen column density was not included as a free parameter in that fit. It was fixed at $1 \times 10^{20} \mathrm{~cm}^{-2}$. They derived a temperature of $T=(5.14 \pm 0.53) \times 10^{6} \mathrm{~K}$ and a bolometric flux of $1.71 \times 10^{-13} \mathrm{erg} \mathrm{cm}^{-2} \mathrm{~s}^{-1}$. The luminosity was calculated for the same distance of $250 \mathrm{pc}$ used by Y94 and it amounts to $1.28 \times 10^{30} \mathrm{erg} \mathrm{s}^{-1}$ in the $0.5-5.0 \mathrm{keV}$ energy range. From those values we can easily see that the emitting area has an even smaller radius than that derived by Y 94 and it is estimated to be about $26 \mathrm{~m}$.

In the timing analysis WH97 extracted events within a 2.5 radius around the X-ray counterpart of PSR B1929+10 from both GIS 2 and 3 instruments with energies between 0.5 and $5.0 \mathrm{keV}$, and obtained 747 counts, 413 of which were estimated to belong to the background. The lightcurve was characterized by a single broad maximum, similar to the ROSAT soft X-ray profile, with a modulation significance of about $3.1 \sigma$, while the pulse fraction was estimated to be $0.35 \pm 0.15(0.5-5 \mathrm{keV})$.

Both groups were unable to discriminate conclusively between a thermal and a non-thermal spectrum, although Y94 as well as WH97 favored the blackbody model. Even though a thermal model provided better overall fits, the inferred emitting areas were surprisingly small.

In this study we revisited the ROSAT PSPC-B and ASCA analyses. Moreover, we also included ROSAT HRI data from a combination of 3 observations lasting $346 \mathrm{ks}$ in total, together with data from a $30 \mathrm{ks}$ RXTE exposure. The main goal was to characterize the timing and spectral properties of PSR B1929+10 in more detail using all available X-ray data. 


\section{Observations}

ROSAT, the Röntgen Satellite, observed PSR B1929+10 as a prime target several times during its mission lifetime with either the PSPC (Position Sensitive Proportional Counter) or with the HRI (High-Resolution Imager) in the focal plane. In this study we used all available ROSAT data i.e. observations with the PSPC-B (30 March 1991-25 April 1991; 43.6 ks exposure) and with the HRI, which pointed three times at PSR B1929+10 for a total combined exposure of $346.7 \mathrm{ks}$. These HRI acquisitions were taken between 9th October and 7th November 1995 for $104.1 \mathrm{ks}$, between 2nd and 21st October 1996 for $136.6 \mathrm{ks}$, and between 13th and 28th April 1997 for $106.0 \mathrm{ks}$. The standard correlation between the ROSAT spacecraft clock and UTC does not permit absolute timing of the events down to millisecond accuracy (see appendix).

Two more datasets for PSR B1929+10 were available, namely from the Advanced Satellite for Cosmology and Astrophysics (ASCA) and the Rossi X-ray Timing Explorer (RXTE). Our target was observed by ASCA between 17th and 19th October 1994 with exposure times of 56.4 and $53.3 \mathrm{ks}$ for the GIS (Gas Imaging Spectrometer) and the SIS (Solidstate Imaging Spectrometer), respectively. To support timing analyses at millisecond scales only data from the high and medium rate telemetry modes of GIS can be used. From RXTE we used PCA (Proportional Counter Array) data taken on 21 th November 1997. The instrument operated in Good Xenon mode for $29.6 \mathrm{ks}$. PCA comprises five identical co-aligned gasfilled proportional counter modules. Each module is referred to as a PCU (Proportional Counter Unit), numbered from 0 to 4. The best time resolution obtained with RXTE is $5 \mu$ s.

\section{Timing analysis}

\subsection{ROSAT PSPC-B}

Point-like X-ray emission was clearly detected from the vicinity of the radio position of the pulsar $\left(\alpha_{2000}=19^{\mathrm{h}} 32^{\mathrm{m}} 13^{\mathrm{s}} .922\right.$, $\delta_{2000}=10^{\circ} 59^{\prime} 32^{\prime \prime}$.07; Pavlov et al. 1996) on the ROSAT PSPC-B Maximum Likelihood Ratio map (MLR map, see e.g. Kuiper et al. 1998). The numbers of source and background counts are derived simultaneously by this method. In the timing analysis we extracted events within an optimum radius (the radius where the signal-to-noise ratio $\mathrm{S} / \mathrm{N}$ maximizes) of $50^{\prime \prime}$ from the X-ray centroid. The timetags of the 604 events (energy range $0.1-2.5 \mathrm{keV}$ ) falling within this radius have subsequently been barycentered and folded using appropriate pulsar parameters (see Table 1 for the ephemeris and general characteristics of PSR B1929+10) to obtain the pulse-phase distribution shown in the left panel of Fig. 1. The profile is characterized by one broad maximum and deviates from a statistically flat distribution at a $4.2 \sigma$ level applying a $Z_{1}^{1}$ method (Buccheri et al. 1983). The sinusoidal shape of $X$-ray pulse profile is in sharp contrast to the strongly peaked radio profile.

In order to quantify the pulsed fraction we have applied two methods, one based on bootstrapping (Swanepoel et al. 1996) and another based on sinusoid fitting. For broad sinusoidal profiles it is expected that the bootstrap method will systematically underestimate the genuine pulsed fraction because parts of the
Table 1. Pulsar characteristics of PSR B1929+10 (PSR J1932+1059).

\begin{tabular}{ll}
\hline \hline Parameter $^{a}$ & Value \\
\hline Right Ascension (J2000) & $19^{\mathrm{h}} 32^{\mathrm{m}} 13^{\mathrm{s}} .855$ \\
Declination (J2000) & $10^{\circ} 59^{\prime} 32^{\prime \prime} .41$ \\
Validity range (MJD) & $48336-48661$ \\
Epoch (MJD) & 48498.000000419 \\
Frequency & $4.4146634668424 \mathrm{~Hz}$ \\
Frequency derivative & $-2.26475 \times 10^{-14} \mathrm{~s}^{-2}$ \\
Age & $3.1 \mathrm{Myr}$ \\
Magnetic field & $0.5 \times 10^{12} \mathrm{G}$ \\
Distance $^{c}$ & $331 \pm 10 \mathrm{pc}$ \\
Spin-down luminosity $^{d}$ & $3.9 \times 10^{33} \mathrm{erg} \mathrm{s}^{-1}$ \\
\hline
\end{tabular}

${ }^{a}$ Ephemeris from D’Amico \& Nicastro (1992) (see http://pulsar.princeton.edu/ftp/gro).

${ }^{b} B=\left(3 I c^{3} P \dot{P} / 8 \pi^{2} R^{6}\right)^{1 / 2}$, for $R=10^{6} \mathrm{~cm}, I=10^{45} \mathrm{~g} \mathrm{~cm}^{2}$.

$c$ From parallax measurements by Brisken et al. (2002).

${ }^{d} \dot{E}=4 \pi^{2} I \dot{P} P^{-3}$, for $I=10^{45} \mathrm{~g} \mathrm{~cm}^{2}$.

leading and trailing wings of the broad profile are considered to be part of the interval used to construct the unpulsed level. Applying the bootstrap method we obtain a pulsed fraction defined as $f_{\text {pulsed }}=\frac{N_{\mathrm{P}}}{N_{\mathrm{P}}+N_{\mathrm{DC}}}$ of $0.24 \pm 0.07$. The number of pulsed counts $N_{\mathrm{P}}$ follows directly from the bootstrap method, while the number of DC source counts $\left(N_{\mathrm{DC}}\right)$ requires an estimate of the number of background counts within the extraction radius of $50^{\prime \prime}$. From the MLR map we could estimate that $140.5 \pm 2.2$ background counts are expected within the extraction radius (see corresponding background level in Fig. 1, left panel).

Because the pulse profile shows one broad maximum, sinusoid fitting using one harmonic provides an adequate description of the measured distribution. Applying sinusoid fitting to the 0.1-2.5 keV PSPC-B pulse profile (see Fig. 3, left panel) we find a pulse fraction of $0.36 \pm 0.08$ which is indeed slightly higher than the bootstrap result, but at the same time still consistent with the value found earlier by Y94 $(0.28 \pm 0.10)$.

By selecting the events further on energy we applied the same sinusoid fitting method to the $0.1-1.28 \mathrm{keV}$ and $1.28-2.5 \mathrm{keV}$ pulse profiles. These two profiles are shown in Fig. 1, middle panel is for $0.1-1.28 \mathrm{keV}$ energy interval and right panel for higher energy range, $1.28-2.5 \mathrm{keV}$. For both lightcurves the modulation significance amounts to $3.0 \sigma$, while the pulsed fractions are $0.33 \pm 0.09$ and $0.42 \pm 0.13$, respectively. These values are the same within their statistical uncertainties. We compared the shapes of the $0.1-1.28 \mathrm{keV}$ and $1.28-2.5 \mathrm{keV}$ lightcurves by applying a $\chi^{2}$-test (Zavlin et al. 1998):

$\chi^{2}=\sum_{i=1}^{N} \frac{\left(m n_{i}-n m_{i}\right)^{2}}{m^{2} n_{i}+n^{2} m_{i}}$,

where $N$ is the number of the phase bins, $n_{i}$ and $m_{i}$ are the numbers of counts in the $i$ th bin for the two lightcurves, and $n$ and $m$ are the sum of $n_{i}$ and $m_{i}$, respectively. This equation implies that the relative errors (e.g., $\sigma_{i}^{(n)} / n_{i}$ ) remain constant when the original lightcurves are scaled for comparing their shapes. The analysis showed that for those two lightcurves $\chi^{2}=17.66$ 

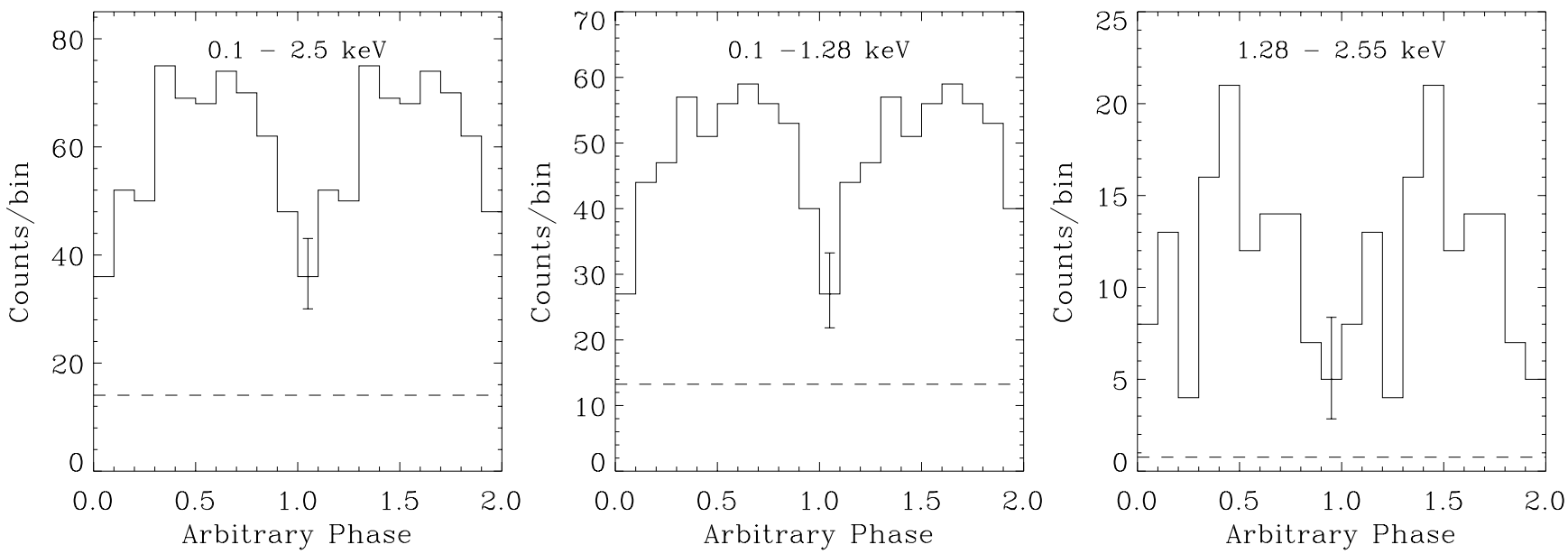

Fig. 1. PSR B1929+10 phase histograms from ROSAT PSPC-B data in three energy ranges, left panel: $0.1-2.5 \mathrm{keV}$, center panel: $0.1-1.28 \mathrm{keV}$, right panel: $1.28-2.5 \mathrm{keV}$. Two cycles are shown for clarity. A typical error bar is shown on each panel. The significances for deviations from statistically flat distributions are $4.15 \sigma$ (604 events), $3.0 \sigma$ (490 events), $3.0 \sigma$ (114 events), respectively. The background levels, as determined from spatial analyses, are presented as dashed lines.
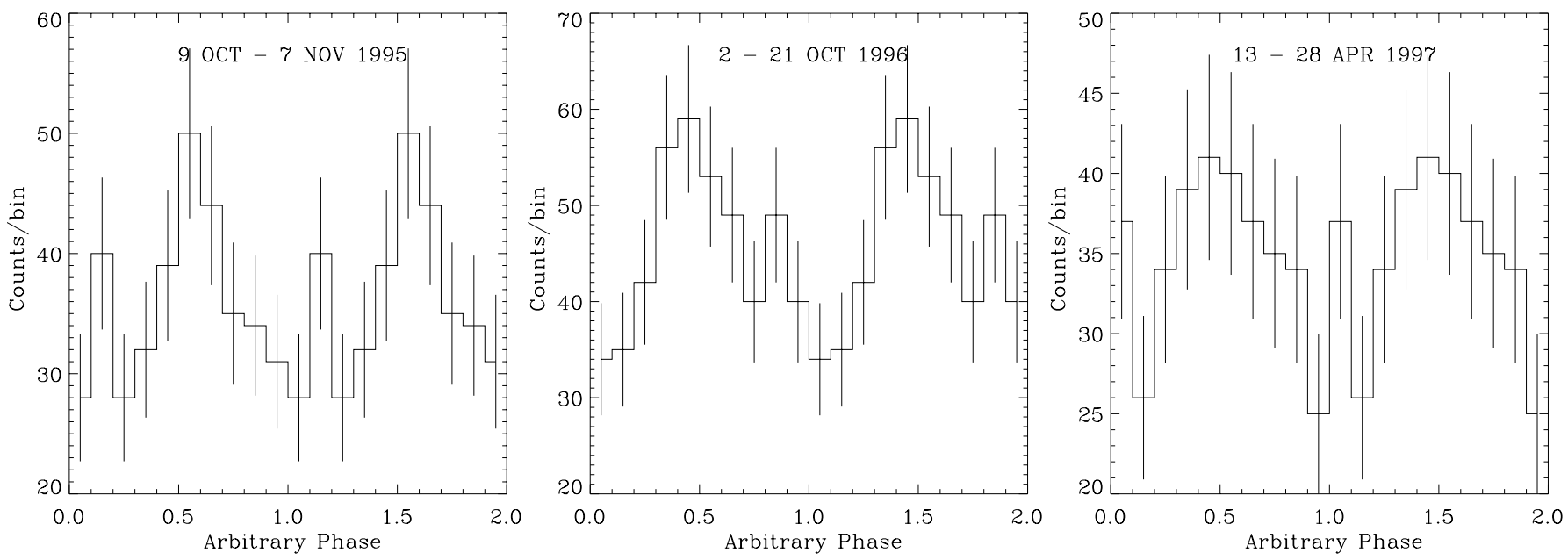

Fig. 2. PSR B1929+10 phase histograms from ROSAT HRI data for three different acquisitions of which the observing time span is given near the top of each panel. Two cycles are shown for clarity. The significance for a deviation from a statistically flat distribution is about $2 \sigma$ for each of these three datasets.

for 9 degrees of freedom. Hence, the reduced $\chi^{2}$ of $1.96 \mathrm{im}-$ plies the possibility that the pulse shape changes with energy ( $\$ 3 \%$ probability for being the same). To confirm this we have to wait for the upcoming results from a recently performed XMM-Newton observation.

In parallel, a similar behaviour has been observed for the other old pulsar, PSR B0950+08 emitting pulsed X-rays by Zavlin \& Pavlov (2004 hereafter ZP04), using the latest XMM-Newton data. They showed that for this pulsar both the pulse shape and the pulsed fraction change with energy. In their work the lightcurve of PSR B0950+08 is presented in four energy bands: $0.2-0.5,0.5-1.0,1.0-5.0$ and $0.2-5.0 \mathrm{keV}$. The pulsed fractions for these energy ranges are $33 \pm 18 \%$, $60 \pm 13 \%, 57 \pm 13 \%$ and $41 \pm 10 \%$, respectively. For energies above $0.5 \mathrm{keV}$ the pulse profile consists of two relatively narrow peaks with a high pulsed fraction $(\approx 60 \%)$, which points to a non-thermal magnetospherical origin of the X-ray emission. The lower energy lightcurve shows a broad single pulse with a lower pulsed fraction of about 33\%. ZP04 are claiming that this energy dependence can be explained by the presence of another, most likely thermal, component.

\subsection{ROSAT HRI}

For the three long ROSAT HRI observations the timing analysis consisted in extracting the events from a 11" aperture around the centroid of the PSR B1929+10 counterpart and subsequently folding the barycentered event times using an appropriate pulsar ephemeris. The obtained, separately for each observation, pulse profiles (Fig. 2 shows left, middle and right panels for the following time spans of the data acquisitions 9 Oct.-7 Nov. 1995, 2-21 Oct. 1996 and 13-28 Apr. 1997, respectively) have all a low modulation significance, between 2 and $3 \sigma$, although the broad bump can easily be discerned in all three lightcurves. On account of the absolute timing limitations (see appendix) we cross-correlated each of the three 

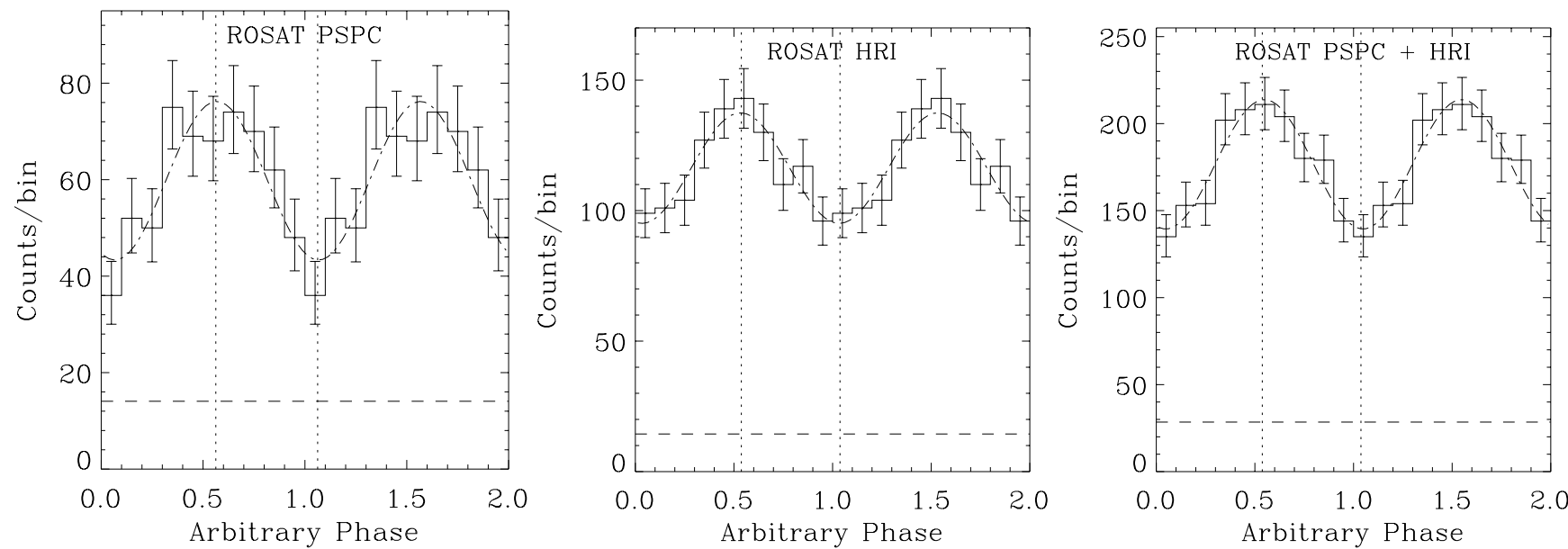

Fig. 3. Light curves for the ROSAT PSPC-B and HRI observations of PSR B1929+10. Left panel: only PSPC-B (0.1-2.5 keV), center panel: only HRI (0.1-2.4 keV), right panel: combined PSPC-B and HRI. Two cycles are shown for clarity. Error bars are shown in each panel. The significances for deviations from statistically flat distributions are $4.15 \sigma, 3.7 \sigma$ and $5.6 \sigma$, respectively. The dashed horizontal lines indicate the background levels determined from spatial analyses. The vertical dotted lines correspond to minimum and maximum phases of the sinusoid fits (the dot-dashed curves).
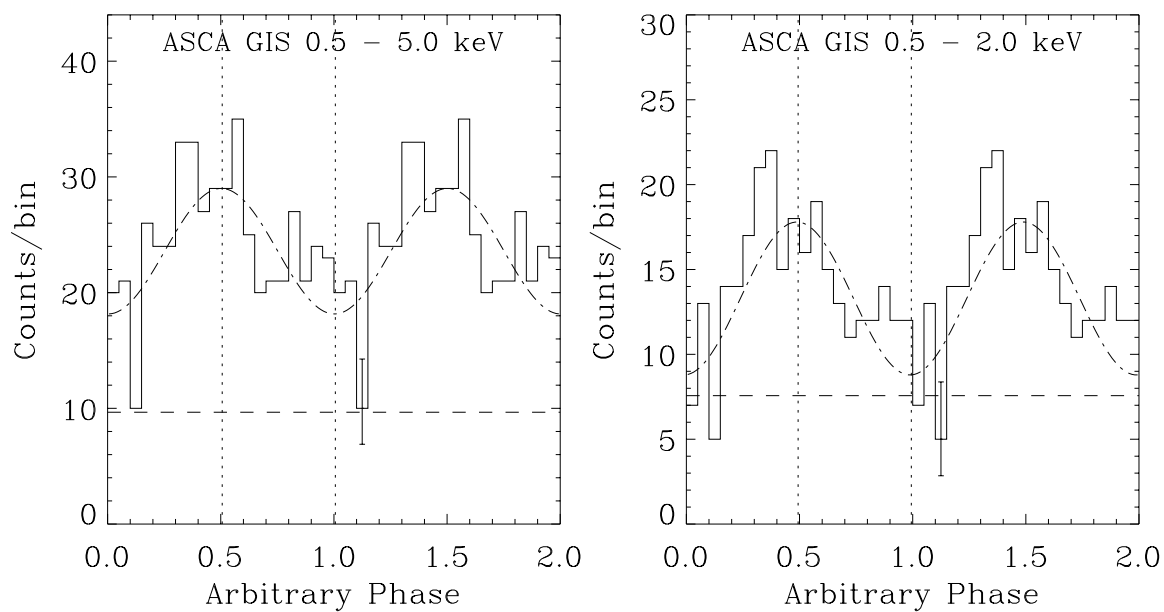

Fig. 4. The ASCA profiles of PSR B1929+10 in two energy intervals: left panel $0.5-5.0 \mathrm{keV}$ (significance $2.7 \sigma$, 493 events) and right panel 0.5-2.0 keV (significance 3.1 $\sigma, 282$ events). The dot-dashed curves indicate the sinusoid fitted to the data, while the vertical dotted lines correspond to the minimum and maximum phases of the fits. In both panels the horizontal dashed lines indicate the background levels determined from spatial analyses. A typical error bar is shown in each panel. Two cycles are shown for clarity.

HRI profiles with the $0.1-2.5 \mathrm{keV}$ PSPC-B profile. In the cross-correlation procedure we used the lightcurves binned into 360 bins. This means that the width of a single bin corresponds to $629 \mu \mathrm{s}$. The obtained shift values were as follow $0.615,0.395$ and 0.525 for the first, second, and third HRI acquisitions, respectively. After applying the phase shifts the combination of the three separate profiles yielded a phase distribution which deviates from a flat distribution at the $3.7 \sigma$ level (Fig. 3, middle panel $)^{1}$. In this case the sinusoid fitting method yielded a pulsed fraction of $0.21 \pm 0.05$, lower, but still consistent with the PSPC-B value. The combined ROSAT PSPC-B and HRI profile is shown in the right panel of Fig. 3, and it deviates from being flat at a $5.6 \sigma$ level. The pulsed fraction of this profile amounts to $0.25 \pm 0.04$.

\footnotetext{
${ }^{1}$ See the appendix for the results from a different approach involving event TDB timestamp folding using just one single ephemeris for the combination of the three HRI observations.
}

\subsection{ASCA GIS}

Of the two detector systems aboard ASCA only the GIS detectors have sufficient timing resolution to support timing analyses at millisecond scales. Data from the high and medium rate telemetry modes were used. The $3.9 \mathrm{~ms}$ timing resolution valid for these rates was sufficiently accurate to sample the pulse period of $227 \mathrm{~ms}$ (see Table 1). In the spatial analysis a Maximum Likelihood method, similar to that used for the ROSAT PSPC-B and HRI data, provided the optimum position for the X-ray counterpart of PSR B1929+10. An extraction radius of $130^{\prime \prime}$ from this position was found to optimize the $\mathrm{S} / \mathrm{N}$ ratio. In the $0.5-5 \mathrm{keV}$ energy interval, the same as used by WH97, 493 event time tags were barycentered and subsequently folded through appropriate timing parameters to yield the corresponding pulse profile. This profile is shown in Fig. 4 left panel and deviates from uniformity at a $2.7 \sigma$ level. The same broad enhancement is visible as in 

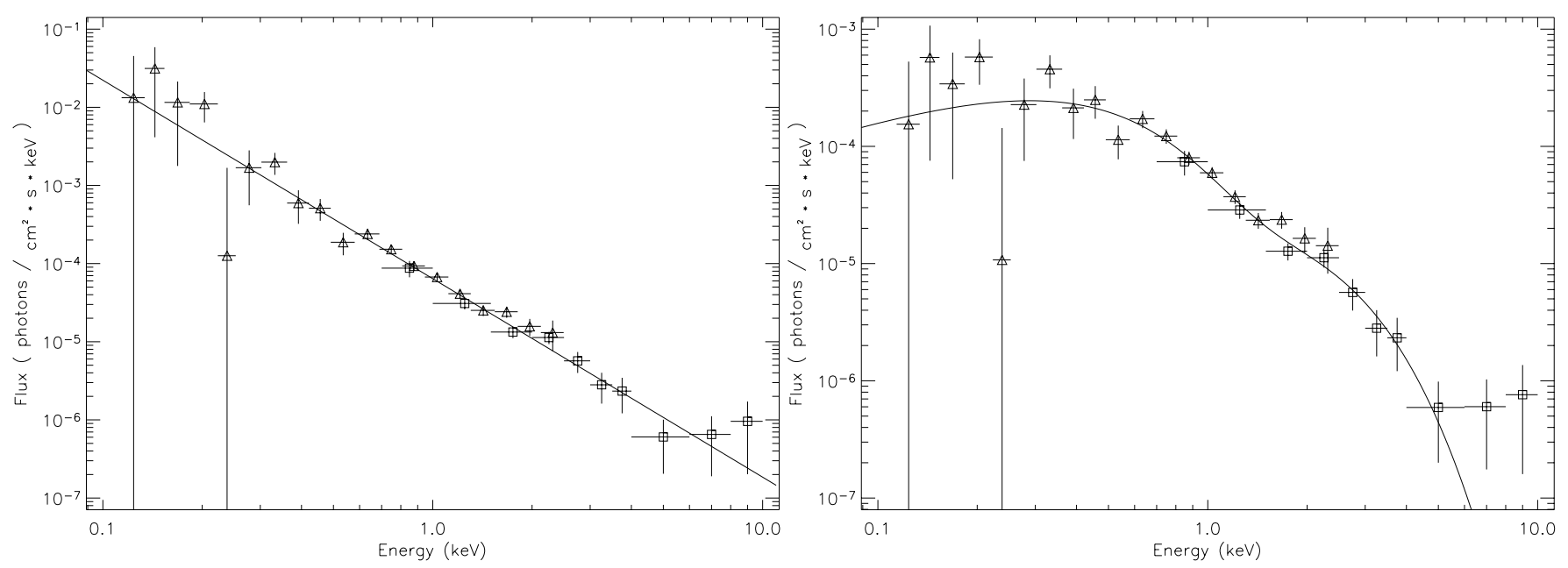

Fig. 5. 0.1-10 keV spectrum of PSR B1929+10 from combined ROSAT PSPC-B (open triangles) and ASCA GIS 2+3 (open squares) data: left panel an absorbed single power-law model, right panel an absorbed double black-body model.

the ROSAT profiles at softer X-ray energies. Constraining the energy to the $0.5-2.0 \mathrm{keV}$ interval (282 events), fully overlapping the ROSAT energy window, yielded a significance of $3.1 \sigma$ (see Fig. 4 right panel). Note, that in the $2.0-5.0 \mathrm{keV}$ energy range (211 events) the profile is consistent with being flat, which means that the main contribution to the pulsed fraction comes from photons with energies lower than $2 \mathrm{keV}$. The pulsed fractions derived from sinusoid fitting for the integral (0.5-5 keV) and the ROSAT overlapping (0.5-2.0 keV) energy ranges are $0.36 \pm 0.11$ and $0.69 \pm 0.18$, respectively. The latter value is high, but because of its large error it is still consistent with the ROSAT numbers, especially in comparison with the high pulsed fraction of $1.28-2.55 \mathrm{keV}$ PSPC-B lightcurve $(0.42 \pm 0.13)$.

Moreover, we can compare our results with those for PSR B0950+08 presented by ZP04. In the energy band $0.2-5.0 \mathrm{keV}$, fully overlapping the ASCA band, they got a pulsed fraction of $41 \pm 10 \%$, while for $0.5-1.0 \mathrm{keV}$ band it increased to $60 \%$. Hence, we argue that the overall situation with respect to the energy dependency of the pulsed fraction is very similar for both pulsars.

\subsection{RXTE PCA}

Finally, we analysed RXTE PCA (2-60 keV) data from a 30 ks observation of PSR B1929+10 (Obs. id. 20156; 21-Nov.-1997) obtained in Good Xenon mode, time tagging each event with a $0.9 \mu$ s time resolution. For the timing analysis we used ftools program fasebin to select events from the top layer of each involved PCU, as well as to barycenter the times of the selected events and finally to fold the barycentered event times with an appropriate ephemeris. The ephemeris used in the folding procedure within fasebin is based on radio timing observations of PSR B1929+10 with the 32-m TCfA radiotelescope at Torun (Poland) since the beginning of June 1997. A dual-channel, circular polarization $L$-band receiving system at frequencies around $1.7 \mathrm{GHz}$ and a $2 \times 64 \times 3 \mathrm{MHz}$ pulsar back-end, the Penn State Pulsar Machine 2 (PSPM-2,
Konacki et al. 1999) are used. For analyzing the radio pulsar timing data the TEMPO software package ${ }^{2}$ has been used.

The folding procedure embedded in fasebin resulted in pulse phase histograms for each of the 256 PHA channels. Unfortunately, in none of the PHA channels or channel intervals $(2-4,4-8,8-16,16-32 \mathrm{keV})$ significant pulsed emission has been detected.

\section{Spectral analysis}

In the spectral analysis we first derived the total (=pulsed plus DC) number of counts assigned to the X-ray counterpart of PSR B1929+10 in several narrow energy slices using a spatial (MLR) analysis which takes into account the presence of nearby sources. We applied this approach to the ROSAT PSPC-B (20 energy bins in the interval $0.1-2.5 \mathrm{keV}$ ) and ASCA GIS 2+3 (10 energy bins in the interval $0.5-10 \mathrm{keV}$ ) data. In contrary to WH97 we chose for ASCA the GIS data, because the GIS sensitivity is higher than the SIS sensitivity for energies beyond $\sim 5 \mathrm{keV}$ and because of uncertainties in the SIS efficiency for energies below $1 \mathrm{keV}$. In the combined ROSAT PSPC-B and ASCA GIS 2+3 spectral fits we used the latest upgrades for the response matrices and we took into account the vignetting corrections for the 3.7 off-axis ASCA observation.

The spectral models fitted to the combined data set consist of an absorbed single power-law (PL), an absorbed single black-body, an absorbed black-body plus power-law and an absorbed double black-body (BB). The best fits were obtained for an absorbed single power-law (Fig. 5, left panel) and an absorbed double black-body model (Fig. 5, right panel). The fit results for these two cases, each with a free hydrogen column density, are shown in Table 2.

If the observed X-ray emission is interpreted in the case of the non-thermal PL model, then the observed $0.1-10 \mathrm{keV}$ flux $(f)$ amounts to $(6.05 \pm 0.49) \times 10^{-13} \mathrm{erg} \mathrm{cm}^{-2} \mathrm{~s}^{-1}$. The derived flux $f$ together with a distance $d$ of $331 \pm 10$ pc adopted from Brisken et al. (2002) implies an isotropic 0.1-10 keV X-ray

\footnotetext{
${ }^{2}$ http://pulsar.princeton.edu/tempo
} 
Table 2. Fits to the combined ROSAT and ASCA spectrum in the energy range $0.1-10 \mathrm{keV}$.

\begin{tabular}{|c|c|}
\hline Parameter & Power law fit \\
\hline$C^{a}$ & $6.41_{-0.4}^{+0.4} \times 10^{-5}$ \\
\hline$\alpha$ & $2.54_{-0.13}^{+0.12}$ \\
\hline$N_{\mathrm{H}}$ & $9.8_{-1.0}^{+1.4} \times 10^{20} \mathrm{~cm}^{-2}$ \\
\hline$\chi_{v}^{2} / v$ & $1.146 / 27$ \\
\hline Parameter & Double black-body fit \\
\hline$C_{1}^{b}$ & $0.0117_{-0.0018}^{+0.0017}$ \\
\hline$k T_{1}$ & $0.176_{-0.004}^{+0.004} \mathrm{keV}$ \\
\hline$C_{2}^{b}$ & $7.879_{-1.26}^{+1.02} \times 10^{-5}$ \\
\hline$k T_{2}$ & $0.595_{-0.03}^{+0.02} \mathrm{keV}$ \\
\hline$N_{\mathrm{H}}$ & $4.43_{-1.12}^{+2.08} \times 10^{20} \mathrm{~cm}^{-2}$ \\
\hline$\chi_{v}^{2} / v$ & $1.132 / 25$ \\
\hline
\end{tabular}

luminosity, $L_{\mathrm{X}},(0.1-10 \mathrm{keV})=4 \pi d^{2} f$, of $(7.9 \pm 0.8) \times 10^{30} \mathrm{erg} \mathrm{s}^{-1}$. From recent VLBA parallax measurements Chatterjee et al. (2004) obtained an even larger distance of $361_{-8}^{+10} \mathrm{pc}$, which would change our $0.1-10 \mathrm{keV}$ isotropic luminosity to $(9.4 \pm$ $0.9) \times 10^{30} \mathrm{erg} \mathrm{s}^{-1}$. The $0.1-10 \mathrm{keV}$ isotropic $\mathrm{X}$-ray luminosity represents $2 \times 10^{-3}$ of the spin-down power $\dot{E}$ (see Table 1) of PSR B1929+10 for the 331 pc distance estimate. For the $0.1-2.4 \mathrm{keV}$ band we obtain an isotropic X-ray luminosity of $(7.1 \pm 0.7) \times 10^{30} \mathrm{erg} \mathrm{s}^{-1}$ which translates to $L_{\mathrm{X}},(0.1-2.4 \mathrm{keV}) / \dot{E}$ ratio of $1.8 \times 10^{-3}$. This value is compatible with e.g. the $\left(L_{\mathrm{X}},(0.1-2.4 \mathrm{keV}), \dot{E}\right)$ relation found by Seward et al. (1988) and later confirmed by Verbunt et al. (1996).

In the case of the double black-body model we are dealing with two different thermal $\mathrm{X}$-ray components with temperatures $2.0_{-0.05}^{+0.05} \times 10^{6} \mathrm{~K}$ and $6.9_{-0.35}^{+0.23} \times 10^{6} \mathrm{~K}$ for $T_{1}$ and $T_{2}$, respectively. For the characteristic age of PSR B1929+10 $(\tau \simeq 3.1 \mathrm{Myr})$ cooling models predict a surface temperature of the neutron star to be $\leq 10^{5} \mathrm{~K}$, much too low to be responsible for the observed X-ray spectrum of PSR B1929+10. However, a plausible explanation could be that the observed $\mathrm{X}$-ray radiation originates from a heated polar cap characterized by two different thermal components (see e.g. Cheng \& Zhang 1999). The simultaneous double BB fit to the ROSAT and ASCA data implies emission luminosities of $L_{1}=(1.56 \pm$ $0.25) \times 10^{30} \mathrm{erg} \mathrm{s}^{-1}$ and $L_{2}=(1.47 \pm 0.25) \times 10^{29} \mathrm{erg} \mathrm{s}^{-1}$ for $T_{1}$ and $T_{2}$ components, respectively. The derived luminosities allow us to calculate the areas $\mathrm{A}$ or the radius $r_{x_{\mathrm{obs}}}$ of the emitting regions responsible for the detected X-rays. Following the simple estimations for the area $A=L_{X} / \sigma T^{4}$ or for the radius $r_{x_{\mathrm{obs}}}=\left(L_{\mathrm{X}} / 4 \pi \sigma T^{4}\right)^{1 / 2}$ we obtain the following numbers: $A_{1}=1.72 \times 10^{9} \mathrm{~cm}^{2}, r_{x_{\mathrm{obs}}, 1}=117 \mathrm{~m}, A_{2}=1.13 \times 10^{6} \mathrm{~cm}^{2}$ and $r_{x_{\mathrm{obs}}, 2}=3 \mathrm{~m}$ for the $2 \mathrm{MK}$ and $7 \mathrm{MK}$ black-body components, respectively. The derived areas correspond to only $\sim 1.4 \times 10^{-4}$ and $\sim 9 \times 10^{-8}$ of the stellar surface, assuming $R_{\mathrm{NS}}=10 \mathrm{~km}$.

The $A_{1}$ and $A_{2}$ values as measured at the NS surface have to be corrected for the gravitational redshift $\left(g_{r}\right)$ relating the areas derived from observations and the intrinsic emitting areas on the star, $4 \pi r_{\text {int }}^{2}$. The gravitational redshift is described by the relation

$g_{r}=(1+z)^{-1}=\left[1-\frac{2 G M}{c^{2} R}\right]^{1 / 2}$,

and for the canonical values $M=1.4 M_{\odot}$ and $R_{\mathrm{NS}}=10 \mathrm{~km}$ it amounts to 0.766. Because $r_{\text {int }}=g_{r} r_{x, \text { obs }}$ then $A_{\text {int }}=A g_{r}^{2}$, and we obtained $A_{\text {int, } 1}=1.01 \times 10^{9} \mathrm{~cm}^{2}$ and $A_{\text {int, } 2}=6.69 \times 10^{5} \mathrm{~cm}^{2}$.

The derived $A_{\text {int, } 1}$, and $A_{\text {int,2 }}$ values are smaller by only a factor of 3 in the low-temperature component case, and significantly smaller $\left(\sim 2 \times 10^{-4}\right)$ for the high-temperature component than the canonical magnetic polar cap defined by the locus of the last closed dipole field line. For the parameters of PSR B1929+10 the cap area is

$A_{p c}=\frac{2 \pi^{2} R_{\mathrm{NS}}^{3}}{c P}=2.9 \times 10^{9} \mathrm{~cm}^{2}$,

where $P$ is the pulsar period, and $R_{\mathrm{NS}}$ was assumed to be $10 \mathrm{~km}$. This shows that for thermal emission model, the X-ray emission area of PSR B1929+10 is roughly consistent with the conventional size of a heated polar cap. The discrepancy of the factor of 3 can be realistic if we consider the complicated distribution of the magnetic field on the surface of a neutron star, distortion of the surface black-body spectrum due to propagation of the radiation through a pulsar magnetosphere and the beam solid angle. The results mean also that the observed X-ray emission does not have its origin in the cooling emission from the entire surface of the neutron star. It is impossible because the implied temperature is unexpectedly high for such an old pulsar and because the emitting area would be unrealisticly small.

\section{1. $N_{\mathrm{H}}$ discussion}

The $N_{\mathrm{H}}$ value obtained from the double BB fit is about twice and that obtained from the PL-fit about six times larger that the $N_{\mathrm{H}}$ value used by Y94 and WH97. In both cases the newly derived column densities are consistent with the updated (radio) distance determined by Brisken et al. (2002) from VLBA data, and the hydrogen distribution measured in the direction of the pulsar (Frisch \& York 1983). Additionally, as Pavlov et al. (1996) have already mentioned, values of $N_{\mathrm{H}}$ obtained from the direct measurements of stars in the neighbourhood of the pulsar are rather controversial. They differ from $\lesssim 10^{19}$ up to $10^{21} \mathrm{~cm}^{-2}$. This behaviour is illustrated in Fig. 6. The presented data, gathered in the Table 3, were obtained using the ISM Hydrogen Column Density Search Tool ${ }^{3}$ available on the Extreme Ultraviolet Explorer (EUVE) web page. For further discussion we will concentrate on only five out of the ten selected sources from the EUVE database nearest to PSR B1929+10. These are: $\sigma$ Aql, $\epsilon$ Del, $o$ Her, WD1910+047 and ES Vul. The first two sources are approximately halfway between the Earth and the pulsar, whereas the third and fourth source are at a two-third way and the last source is about $80 \mathrm{pc}$ farther from the observer than the pulsar is. The dependence of

\footnotetext{
${ }^{3}$ http://stdatu.stsci.edu/euve/ism/ismform.html
} 

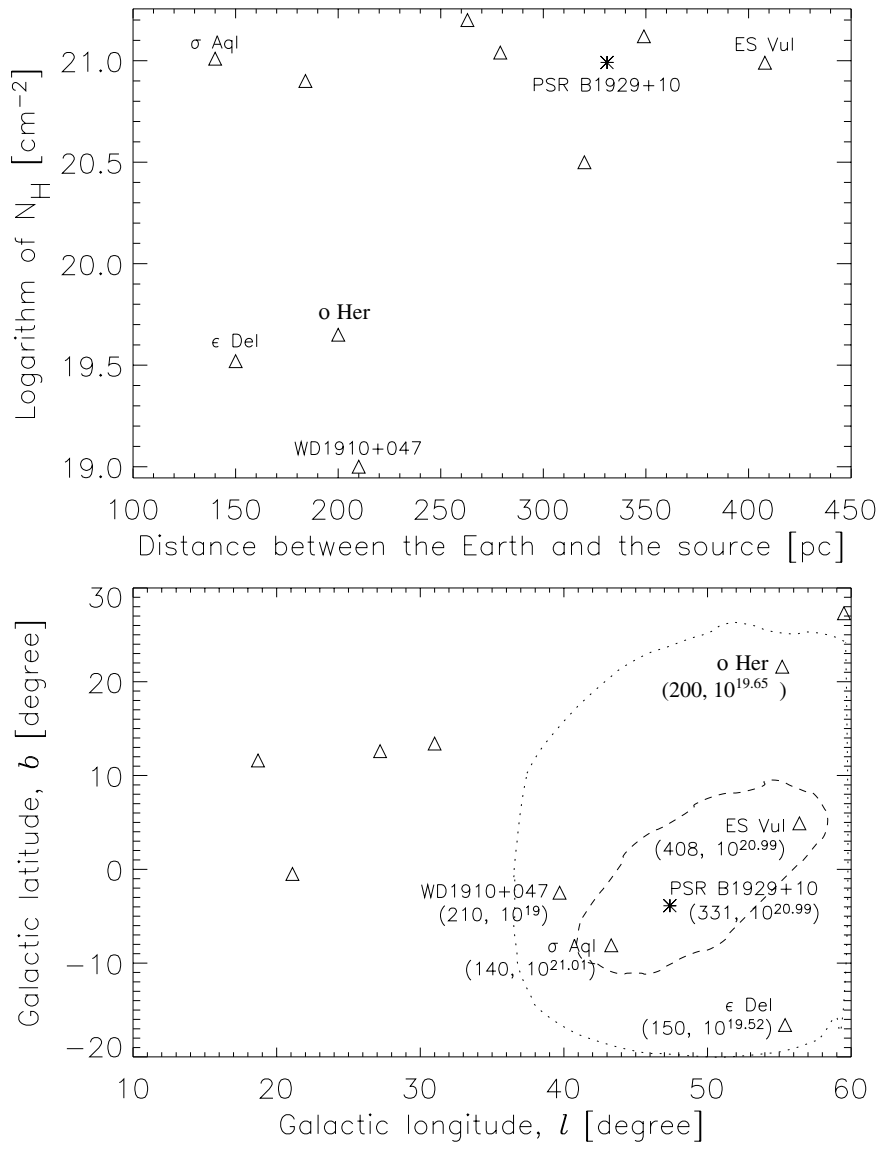

Fig. 6. Upper panel: the dependence of the logarithm of the interstellar hydrogen column densities on the distances for ten stars from the EUVE database and the pulsar. For the discussion only five stars were selected and they are labeled in the figure. In the bottom panel the spatial distribution of the mentioned sources in the Galactic coordinates $l, b$ is shown. The stars of interest and the pulsar are labeled. Additionally, the distance in parsecs and the $N_{\mathrm{H}}$ value in $\mathrm{cm}^{-2}$ for each object is given in brackets. Dotted and dashed lines encircle two regions with different mean $N_{\mathrm{H}}$ values, $2.4 \times 10^{19} \mathrm{~cm}^{-2}$ for the more extended region and $9.92 \times 10^{20} \mathrm{~cm}^{-2}$ for the smaller one.

the logarithm of the interstellar hydrogen column densities on the distances for all ten sources, as well as for PSR B1929+10, is shown in the upper panel of Fig. 6. It is quite clear, that in some cases, even if the source is not very far away the logarithm of $N_{\mathrm{H}}$ can be as large as 21 , as it happens for example for $\sigma$ Aql. On the contrary, $\epsilon$ Del being roughly on the same distance as $\sigma$ Aql, or $o$ Her and WD1910+047 distant only about $50 \mathrm{pc}$ from $\sigma$ Aql all do have rather small values of the logarithm of $N_{\mathrm{H}}$. This suggests that the interstellar medium is very patchy in that direction. The spatial distribution of all sources in the Galactic coordinates $(l, b)$ is shown in the bottom panel of Fig. 6 . For the pulsar and the five chosen stars their names and the values of their distances and $N_{\mathrm{H}}$ (in brackets) are presented. We can distinguish two regions. The first region encircles the surface with borders determined by the positions of the following sources WD1910+047, $\epsilon$ Del and $o$ Her. For this region, which is placed around $150-200$ pc away, the average value of neutral hydrogen column density is at the level of $2.4 \times 10^{19} \mathrm{~cm}^{-2}$. The second region, overlapping the first
Table 3. The parameters of ten EUVE sources placed in the pulsar direction as derived by the ISM Hydrogen Column Density Search Tool.

\begin{tabular}{cccccc}
\hline \hline HD name & Name & $l$ & $b$ & $d^{a}$ & $N_{\mathrm{H}}^{b}$ \\
\hline HD 180968 & ES Vul & 56.4 & 4.9 & 408.0 & $20.99^{\star}$ \\
- & WD1910+047 & 39.7 & -2.5 & 210.0 & $19.00^{\dagger}$ \\
HD 170740 & - & 21.1 & -0.5 & 279.0 & $21.04^{\star}$ \\
HD 163472 & V2052 Oph & 27.2 & 12.6 & 349.0 & $21.12^{\star}$ \\
HD 166014 & $o$ Her & 55.2 & 21.6 & 200.0 & $19.65^{\ddagger}$ \\
HD 161056 & - & 18.7 & 11.6 & 263.0 & $21.20^{\star}$ \\
HD 164284 & 66 Oph & 31.0 & 13.4 & 184.0 & $20.90^{\star}$ \\
HD 162094 & - & 59.5 & 27.3 & 320.0 & $20.50^{\star}$ \\
HD 195810 & $\epsilon$ Del & 55.4 & -16.6 & 150.0 & $19.52^{\ddagger}$ \\
HD 185507 & $\sigma$ Aql & 43.3 & -8.1 & 140.0 & $21.01^{\star}$ \\
\hline
\end{tabular}

a Distance (in parsecs) between the star and the Earth.

${ }^{b}$ Logarithm of $N_{\mathrm{H}}$ (in $\mathrm{cm}^{-2}$ ), reference ${ }^{\star}$ Diplas \& Savage (1994);

${ }^{\dagger}$ Paerels \& Heise (1989); ${ }^{\star}$ Welsh et al. (1991); ${ }^{\star}$ Bohlin et al. (1983); * Brown et al. (1991).

one, seems to be much smaller than that and elongates along the line connecting $\sigma$ Aql, PSR B1929+10 and ES Vul. The average $N_{\mathrm{H}}$ is at the level of $9.92 \times 10^{20} \mathrm{~cm}^{-2}$ here and it is about 40 times larger than that of region one.

From the spatial distribution of the interstellar extinction (see Neckel \& Klare 1980) we were able to reconstruct similar values of $N_{\mathrm{H}}$ for the described stars. For this purpose we first used the general maps to identify our field of interest designated after Neckel \& Klare by the running numbers 50/-5.0 for PSR B1929+10, 52/0.0 for ES Vul and 42/-4.0 for WD1910+047 and $\sigma$ Aql. (Because the maps were available only for the galactic belt, $\epsilon$ Del was out of range). Subsequently, from the $A_{\mathrm{V}}(r)$ diagrams of the mentioned regions and by using the following relations $R=\frac{A_{\mathrm{V}}}{E_{(B-V)}}$ and $\frac{N_{\mathrm{H}}}{E_{(B-V)}}=4.8 \times 10^{21}$ atoms cm${ }^{-2} \mathrm{mag}^{-1}$ (Bohlin et al. 1978) we obtained the following values of $N_{\mathrm{H}}, 1.03 \times 10^{21}, 1.26 \times 10^{21}$, $3.25 \times 10^{20}$ and $2.17 \times 10^{21}$ atoms cm ${ }^{-2}$ for PSR B1929+10, ES Vul, WD1910+047, $\sigma$ Aql, respectively. One can notice that a significant disagreement occurs only in the case of WD1910+047. The reason of this is that WD1910+047 is near the border of the region 42/-4.0 and moreover the relation $A_{\mathrm{V}}(r)$ is not very well depicted for this region.

Finally, our results are supported by outcomes from a numerical algorithm developed by Hakkila et al. (1997) calculating three-dimensionally the visual interstellar extinction and its error from the galactic longitude, galactic latitude and distance. After using their extinction code for the pulsar parameters $(l, b$, d) we obtained a visual interstellar extinction $A_{\mathrm{V}}$ of 0.55 and error $\sigma_{A_{\mathrm{V}}}$ of 0.27 which results in $E_{B-V}=0.177 \pm 0.087$ and finally $N_{\mathrm{H}}=(8.5 \pm 4.2) \times 10^{20} \mathrm{~cm}^{-2}$, which is fully consistent with our values of $N_{\mathrm{H}}$ based on PL and double BB fits.

At last we would like to mention that all the data collected on the EUVE server come from the database published in The Distribution of Neutral Hydrogen in the Interstellar Medium (Fruscione et al. 1994), plus some additional new measurements from Diplas \& Savage (1994). 


\section{Summary}

Our revisited analysis of X-ray data of PSR B1929+10 from ROSAT, ASCA and RXTE observations yielded an improved timing signal at soft X-rays of $5.6 \sigma$ for the combined ROSAT PSPC-B/HRI pulse profile, and a non-detection for energies beyond $\sim 2 \mathrm{keV}$ (RXTE PCA). Analyzing the pulse profiles at soft X-rays (ROSAT PSPC-B) in two different energy bands yielded the pulsed fractions $0.33 \pm 0.09$ in the $0.1-1.28 \mathrm{keV}$ band, $0.42 \pm 0.13$ in the $1.28-2.5 \mathrm{keV}$ band, and $0.69 \pm 0.18$ for the $0.5-2 \mathrm{keV}$ ASCA GIS band.

A spectral analysis of the combined ROSAT/ASCA data in the $0.1-10 \mathrm{keV}$ energy range indicated that both an absorbed power-law (non-thermal) model and an absorbed double blackbody model provide an adequate description of the observed $\mathrm{X}$-ray spectrum. For the double BB model a plausible explanation could be that the observed X-ray radiation originates from a heated polar cap characterized by two different thermal components (Cheng \& Zhang 1999). The derived hydrogen column densities $\left(N_{\mathrm{H}}\right)$ are in both cases much larger than the value used in previous X-ray studies. However, the newly determined $N_{\mathrm{H}}$ values are more consistent with the updated (increased) distance to PSR B1929+10 in combination with the measured hydrogen distribution in the direction of the pulsar.

An observation performed with the XMM-Newton satellite could confirm our results and the improved statistics would help to fix the column density $N_{\mathrm{H}}$ and to discriminate conclusively between thermal and non-thermal models for the X-ray emission from this pulsar.

Acknowledgements. This research was supported by KBN grant PBZ-KBN-054/P03/2001 and Polish Foundation of Astronomy. Aga Słowikowska acknowledges the staff of SRON for their hospitality during her stay there, when most of this work was done. She would like to thank Bronek Rudak who developed the idea for this paper. Thanks also to Roberto Mignani and Ryszard Szczerba for the discussions about the ISM. We acknowledge Michael Kramer for providing the Jodrell Bank TOAs of PSR B1929+10 over the time spans when the ROSAT observations of this pulsar were taken. We are pleased to acknowledge Dr. J. Halpern for his referee comments.

\section{Appendix A: ROSAT timing}

Timing analysis with the ROSAT PSPC and HRI instruments at millisecond accuracies is certainly no trivial task. Several authors analysing different fast spin-down powered pulsars encountered severe problems in attempts to phase align the ROSAT profiles with profiles obtained at different wavelengths or to phase align ROSAT profiles from different ROSAT observations.

For instance, in Greiveldinger et al. (1995) the pulse maximum in the $0.1-2.4 \mathrm{keV}$ range for the $150 \mathrm{~ms}$ pulsar PSR $\mathrm{B} 1509-58$ was found at $0.13 \pm 0.05$ with respect to the radio pulse, while it is nowadays known that the pulse maximum occurs between 0.27-0.30 (pulse shape is weakly energy dependent) for energies in excess of $\sim 0.5 \mathrm{keV}$ (see e.g. Kuiper et al. 1999, and references therein for the ASCA 0.7-2.2 keV profile overlapping the ROSAT energy band). Furthermore, Seward et al. (2000) reported radio/soft X-ray alignment problems for the Vela pulsar (89 ms) analysing ROSAT HRI data, which manifest theirselves as absolute phase errors of about 0.2 . In this case the problems are probably related to (an) ignored leap second jump(s) in the spacecraft clock-to-UTC (SCC-TO-UTC) conversion relation (see later this section). Moreover, studying the Geminga pulsar (237 ms) at soft X-rays Halpern \& Wang (1997) reported discrepancies in the phase alignment of different ROSAT Geminga observations, which could be traced back to leap second ignorance in the PROS software. Finally, in Kuiper et al. (2001) a ROSAT HRI 0.1-2.4 keV pulse profile for the Crab pulsar (33 ms) was shown based on a $7.98 \mathrm{ks}$ observation (RH400639N00) performed over the time period from 4-15 March 1995. In this observation four consecutive (ROSAT) orbital intervals could be identified yielding a stable pulse profile. The most dominant chunk showed the Crab first pulse at phase 0.82 (the other chunks are at $0.88,0.86$ and 0.85 , indicating the drift within an observation), while 0.9915 (INTEGRAL/RXTE/Chandra/XMM) is expected. Contrary to the scatter in the phase arrival of the first Crab pulse, which is within the range of scatter of the SCC-TO-UTC correlation, the overall shift of $\sim-0.18(\simeq-6 \mathrm{~ms})$ is hard to explain.

Given these problems we initiated ROSAT timing studies ourselves using fast spin-down powered pulsars with a) a known phase relation to radio frequencies, b) which are sufficiently strong to show a significant timing signal in relatively short observations and c) which have a stable soft $\mathrm{X}$-ray profile over the entire soft X-ray band (i.e. no strong energy-dependent behaviour like that observed e.g. for the Vela and Geminga pulsars, which are moreover also weak $\mathrm{X}$-ray pulsars). The only two sources meeting these criteria are the Crab pulsar (PSR B0531+21) and PSR B1509-58. Our (CGRO-based) timing software used for this purpose has successfully been applied to timing data from a wide variety of high-energy instruments (CGRO EGRET/COMPTEL/BATSE, RXTE PCA/HEXTE, Chandra etc.) and takes leap second jumps correctly into account (verified for e.g. CGRO Crab, Vela etc data). The ROSAT ${ }^{4}$ timing data can easily be processed by this software because the ROSAT SCC timestamps could be converted to UTC timestamps (=default from the CGRO instruments) applying the SCC-TO-UTC time correlation model specified in the ssc-to-utc.tfits file (retrievable from http : / / wave. xray.mpe.mpg.de/rosat/calibration / timing).

For PSR B1509-58 we found 4 different ROSAT observations (RP500041A00/A01; PSPC-Feb. 1991, 1992 resp. and RH400409N00/A01; HRI-Feb./Sep. 1994) useful for absolute timing studies. Phase folding the solar system barycentered TDB (converted from UTC) timestamps of events extracted from circular regions centered on the pulsar position (extraction radii PSPC/HRI are 50" and 12", respectively) with well established/verified pulsar ephemerides from the Princeton and ATNF pulsar databases yielded for all four

\footnotetext{
4 It is interesting to note that the ROSAT mission with a life time of about 8.5 year (1-6-1990-12-2-1999) overlapped 8 time intervals in which new leap seconds were introduced.
} 
observations the pulse maximum of the asymmetric pulse in the range 0.60-0.68.

For the Crab pulsar we selected 6 different PSPC/HRI observations performed between 2-3-1991 and 22-3-1997 encompassing 5 different periods in which leap second adjustments have been applied. These Crab observations were: RF500065N00, PSPC 1.9 ks 2-3-1991; RH141890N00/RH141892N00, HRI 1.4/1.8 ks 16/17-9-1992; RH400639N00 HRI 7.9 ks 4/15-3-1995; RH500464N00 HRI 32.6 ks 9/29-3-1996; and RH400867N00 HRI 33.5 ks 20/22-3-1997. Applying again established/verified Crab pulsar ephemerides (Princeton and Jodrell Bank databases) in the folding process we found the first Crab pulse at arrival phases between $0.83-0.91$ for the 4 observations selected before 31-12-1995, and in the range 0.03-0.10 for the 2 observations selected after 31-12-1995, while an arrival phase of 0.9915 is expected (note that all PSR B1509-58 observations had been performed before 31-12-1995). The Crab first pulse arrival phases of the 2 sets can be made compatible (i.e. within the rms of the SCC-UTC time correlation) by assuming that the leap second insertion at 31-12-1995 was not accounted for in the two observations after this date. Adding, for these two observations, $1 \mathrm{~s}$ to the UTC timestamps derived from the regular SCC-UTC time correlation model put the first Crab pulse in the range 0.94-1.01.

Comparing now the Crab/PSR B1509-58 alignment results for the observations performed before 31-12-1995 the apparent offset of 0.37(4), found for PSR B1509-58 from the established value of 0.27 , can be understood if all four PSR B1509-58 datasets lack the $1 \mathrm{~s}$ addition, i.e. adding $1 \mathrm{~s}$ to the UTC times derived from the regular SCC-UTC correlation puts the maximum of the PSR B1509-58 pulse at $\sim 0.25$. Postulating a minor overall timing correction of about 3-6 ms in addition to this $1 \mathrm{~s}$ put both the Crab and PSR B1509-58 pulses at their expected phase locations (within the rms of the SCC-UTC time correlation).

From this study it should be clear that the direct combination of different ROSAT observations, performed at different widely separated dates crossing leap second insertion dates, can be very tricky in the case of weak pulsars in order to increase the signal-to-noise ratio. Therefore, in the HRI timing study of PSR B1929+10 we decide to combine the separately correlated (with the PSPC profile, see Sect. 3.2.) HRI pulse profiles to obtain the total detection significance of the pulsed signal. Later on we verified this approach by using one single PSR B1929+10 ephemeris, specially prepared from Jodrell Bank TOAs (validity time interval 49927-50595 MJD; reference epoch 50261 MJD TDB), overlapping all three HRI observations in the folding process taking into account that two PSR B1929+10 HRI observations had been performed after 31-12-1995 and one before (and thus $1 \mathrm{~s}$ should be added to the latter to align it with the former; see notes on the Crab timing).

\section{References}

Bohlin, R. C., Savage, B. D., \& Drake, J. F. 1978, ApJ, 224, 132

Bohlin, R. C., Jenkins, E. B., Spitzer, L., et al. 1983, ApJS, 51, 277

Brisken, W. F., Benson, J. M., Goss, W. M., \& Thorsett, S. E. 2002, ApJ, 571, 906

Brown, A., Drake, S. A., van Steenberg, M. E., \& Linsky, J. L. 1991, ApJ, 373, 614

Buccheri, R., Bennett, K., Bignami, G. F., et al. 1983, A\&A, 128, 245

Cheng, K. S., \& Zhang, L. 1999, ApJ, 515, 337

Chatterjee, S., Cordes, J. M., Vlemmings, W. H. T., et al. 2004, ApJ, 604, 339

D’Amico, N., Nicastro, L. 1992, GRO/radio timing data base, Princeton University

Diplas, A., \& Savage, B. D. 1994, ApJS, 93, 211

Frisch, P. C., \& York, D. G. 1983, ApJ, 271, L59

Fruscione, A., Hawkins, I., Jelinsky, P., \& Wiercigroch, A. 1994, ApJS, 94, 127

Greiveldinger, C., Caucino, S., Massaglia, S., Ögelman, H., \& Trussoni, E. 1995, ApJ, 454, 855

Halpern, J. P., \& Wang, F. Y.-H. 1997, ApJ, 477, 905

Hakkila, J., Myers, J. M., Stidham, B. J., \& Hartmann, D. H. 1997, AJ, 114, 2043

Konacki, M., Lewandowski, W., Wolszczan, A., Doroshenko, O., \& Kramer, M. 1999, ApJ, 519, L81

Kuiper, L., Hermsen, W., Bennett, K., et al. 1998, A\&A, 337, 421

Kuiper, L., Hermsen, W., Krijger, J. M., et al. 1999, A\&A, 351, 119

Kuiper, L., Hermsen, W., Cusumano, G., et al. 2001, A\&A, 378, 918

Neckel, T., \& Klare, G. 1980, A\&AS, 42, 251

Paerels, F. B. S., \& Heise, J. 1989, ApJ, 339, 1000

Pavlov, G. G., Stringfellow, G. S., \& Córdova, F. A. 1996, ApJ, 467, 370

Seward, F. D., \& Wang, Z.-R. 1988, ApJ, 332, 199

Seward, F. D., Alpar, M. A., Flanagan, C., et al. 2000, ApJ, 536, 948

Swanepoel, J. W. H., de Beer, C. F., \& Loots, H. 1996, ApJ, 467, 261

Verbunt, F., Kuiper, L., Belloni, T., et al. 1996, A\&A, 311, L9

Wang, F. Y.-H., \& Halpern, J. P. 1997, ApJ, 482, L159

Welsh, B. Y., Vedder, P. W., Vallerga, J. V., \& Craig, N. 1991, ApJ, 381,462

Yancopoulos, S., Hamilton, T. T., \& Helfand, D. J. 1994, ApJ, 429, 832

Zavlin, V. E., \& Pavlov, G. G. 1998, A\&A, 329, 583

Zavlin, V. E., \& Pavlov, G. G. 2004, ApJ, 616, 452 